\title{
CONSTRUCTION OF A PORTABLE MINI LUMINESCENCE- MEASUREMENT SYSTEM EQUIPPED WITH A MINIATURE X-RAY GENERATOR
}

\author{
TOMOYUKI TAKEUCHI ${ }^{1}$, TOMOHIRO SHIBUTANI ${ }^{2}$ and TETSUO HASHIMOTO ${ }^{2}$ \\ ${ }^{1}$ Graduate School of Science and Technology, \\ Niigata University, Niigata 950-2181, Japan \\ ${ }^{2}$ Department of Chemistry, Faculty of Science, \\ Niigata University, Niigata 950-2181, Japan
}

Received 31 January 2007

Accepted 30 January 2008

\begin{abstract}
For the purpose of both luminescence dosimetry and dating, a new portable mini automated luminescence-measuring system has been developed. The main parts of the new system consist of the stimulation unit, an irradiation unit and the photon detector. The size of the main measuring box is approximately $300 \times 300 \times 300 \mathrm{~mm}$. In particular, to keep the electric power as low as possible, a miniature X-ray (Oxford, Eclipse II reflection target) generator and a metal-packaged photomultiplier tube (PMT) (Hamamatsu photonics, H7421-40) were adopted here. 16 blue LEDs were employed for the stimulation of quartz OSL. The same LED holder can be used for pulsed-OSL (P-OSL) measurements. The weight of the main part of this system was about $15 \mathrm{~kg}$.

In order to check the usefulness of the new system, red thermoluminescence (RTL) glow curves were measured to estimate the signal-to-noise ratio ( $\mathrm{S} / \mathrm{N}$ ratio) using a volcanic quartz sample. These results show that the new system improves the $\mathrm{S} / \mathrm{N}$ ratio in comparison with an existing handmade system. The estimated RTL equivalent (or paleo) dose using quartz extracted from an archaeological roof tile of Shin-Yakushiji temple by means of the SAR protocol was in good agreement with previous measurements. This proves that the new system is suitable for practical use for RTL-measurements either for dosimetry or for dating with a high $\mathrm{S} / \mathrm{N}$ ratio.
\end{abstract}

Keywords: TL, pulse OSL, miniature X-ray generator, portable automated luminescence-measuring system.

\section{INTRODUCTION}

When natural or artificial radiation is incident upon insulating crystals, a small proportion of the deposited energy should be stored in the lattice at defect sites. If the irradiated sample is then excited, luminescence phenomena may be observed. Either heat or light may be used for stimulation and the resulting emitted light is termed thermoluminescence (TL) or optically stimulated luminescence (OSL), respectively. The amount of luminescence is found to be related to the accumulated radiation dose since the last event of heating or exposure of sunlight. Therefore, the TL and OSL phenomena are known to be effective for archaeological chronology,

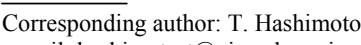

e-mail: hashimoto-t@ginzado.ne.jp

ISSN 1897-1695 (online), 1733-8387 (print) (C) 2008 GADAM Centre, Institute of Physics, Silesian University of Technology.

All rights reserved.
Quaternary geological dating of sediments and retrospective dosimetry using minerals such as quartz and feldspar (Aitken, 1985 and 1998; Bøtter-Jensen et al., 2003). Nowadays, luminescence techniques are recognized to be applicable for dating events over timescales ranging from $10^{2}$ to $10^{6}$ years.

The red TL (RTL) emission from quartz, which possesses a broad emission band with a peak around $630 \mathrm{~nm}$, has been widely observed in grains extracted from volcanic ash layers and burnt materials such as pottery and roof tiles (Hashimoto et al., 1986 and 1997). Additionally, several researchers (Ganzawa et al., 2005; Fattahi and Stokes, 2000) have reported that RTL phenomena are preferable to other luminescence signals for dating of burnt archaeological samples owing to the signal stability over long periods $\left(10^{6}\right.$ years $)$. 
Previously, in the author's laboratory, an automated luminescence-measuring system installed with a small X-ray generator (Varian, VF-50) was constructed (Hashimoto et al., 2002; Nakagawa et al., 2003). However, that luminescence-measurement system is too heavy to carry and requires a large electric power supply over $500 \mathrm{~W}$. Therefore, the use of this system is limited to the dark room of the experimental laboratory associated with large electric power. A portable luminescence-measurement system is useful for field work and as an educational tool and similar. A trial of a portable OSL-measurement system without an irradiation facility has previously been reported by Poolton et al. (1994), but this system too was rather heavy. Here we describe the development of a portable mini automated luminescence-measuring system, which decreases both the size and the weight of the electrical system as well as the electronic power required. In addition, the new system was designed to be able to make pulsed-OSL (P-OSL) measurements in addition to the RTL measurements. This paper describes features of a newly portable mini automated luminescencemeasurement system, and presents the results of some performance tests for RTL and P-OSL measurements using the system.

\section{SYSTEM DESIGN}

Fig. 1 shows a conceptual view of the portable automated mini luminescence-measuring system. A computer controls the system and can be used for the single-aliquot regenerative-dose (SAR) protocol (Murray and Wintle, 2000). The main part of the new system consists of the stimulation unit (a LED holder and heater assembly), an irradiation unit and the luminescence detection system. To minimize the electrical power requirements so that it could in theory be powered by a small battery, a miniature X-ray generator and a metal-packaged PMT were adopted for the new system. The LED holder has 16 blue LEDs (Nichia Chemical Industries Ltd., NSPB-500S) and 16 infrared LEDs (Hamamatsu Photonics, L2690-02). These diodes have peak emission at $470 \mathrm{~nm}$ and $890 \mathrm{~nm}$, respectively, with $50 \mathrm{~nm}$ FWHM value. Eight sample pans on a rotation wheel can be measured in one cycle. The control electronics allow the LEDs to be repeatedly pulsed for pulsed-OSL (P-OSL) measurements, as well as

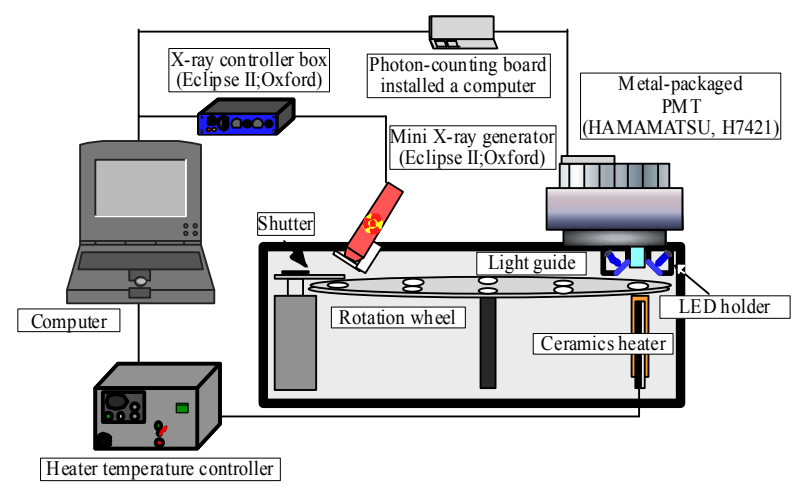

Fig. 1. Conceptual view of the portable mini automated luminescencemeasuring system. for long term illumination for continuous-wave OSL (CW-OSL) measurements. Each feature is described in the following section. Tentatively, it was realized that the total electrical power required is less than $50 \mathrm{~W}$, except for the PC and the heater which required an additional $128 \mathrm{~W}$. The main measuring box size is approximately $300 \times 300 \times 300 \mathrm{~mm}$.

\section{The miniature $\mathrm{X}$-ray generator}

A miniature X-ray generator (Oxford, Eclipse-IIReflection) was employed as an irradiation source. This X-ray generator was of reflection type, so the irradiation facility should be installed at an angle of $28^{\circ}$ as seen in Fig. 1. The total weight of this generator amounts to $330 \mathrm{~g}$, equivalent to about one third of the weight of the $\mathrm{X}$-ray system used previously (Varian, VF-50J) (Yawata and Hashimoto, 2004).

In this new generator, the maximum anode current and voltage are $0.1 \mathrm{~mA}$ and $30 \mathrm{kV}$, respectively, giving a maximum electrical power requirement of $3 \mathrm{~W}$. Since the average X-ray energies from this X-ray generator are about $9 \mathrm{kV}$, the half-thickness layer value of $\mathrm{Al}$ is calculated to be about $0.1 \mathrm{~mm}$ from the mass-attenuation coefficients of X-ray. Thus, Al plate of $0.1 \mathrm{~mm}$ thickness should be sufficient for shielding of low-energy X-rays from this generator enabling the miniaturization of the system. However, for the current model the box was made from steel for convenience. Furthermore, the use of a fullerene as a cathode material in the X-ray tube, gives the possibility of long-term life for the generator.

Since the X-rays arise from collision of the accelerated electrons with the rhodium ( $\mathrm{Rh}$ ) target, the energy spectrum of the X-ray consists mainly of Bremsstrahlung radiation, accompanied with a line spectrum or specific $\mathrm{X}$-ray at about $6 \mathrm{keV}$. The low energy X-rays at $6 \mathrm{keV}$ are too weak to pass though the full diameter of coarse grains due to their short range within quartz (halfthickness layer value for quartz: $22 \mu \mathrm{m}$ ), so that inhomogeneous ionization will occur in the case of a coarse quartz grain. Therefore, to ensure good reproducibility of the dose rate, the X-ray beam was hardened by inserting an Al absorber of $24 \mu \mathrm{m}$ thickness between the X-ray generator and the sample pan.

The dose-rate can be adjusted by changing the applied voltage and/or the current because the X-ray strength is proportional to the anode current and the applied voltage. In Fig. 2, there exists a good linear relationship between current and dose-rate at current beyond $10 \mu \mathrm{A}$. As a result, X-ray dose-rates are obtained over the range 1.3-6.8 Gy/min by varying the anode current from $10-50 \mu \mathrm{A}$ under a fixed anode voltage of $30 \mathrm{kV}$ as tube potential.

\section{The metal-packaged PMT and heater assembly}

The metal-packaged PMT (Hamamatsu photonics, H7421-40) with a GaAsP photocathode surface offers a response from the UV $(300 \mathrm{~nm})$ to the far-red $(720 \mathrm{~nm})$ region, which is particularly suitable for the detection of wide ranges for visible light regions, including red luminescence. Even when no light is incident on the photocathode, a small dark current can arise owing to thermo- 


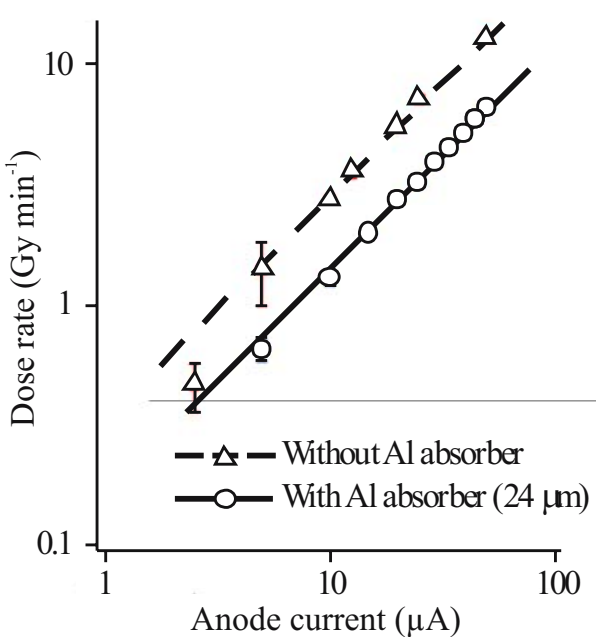

Fig. 2. Plot of the dose rate as a function of the anode current. RTLquartz samples from volcanic origin were used for the measurements. The dose standardization of these curves was carried out using the same quartz grains irradiated to 5 and $10 \mathrm{~Gy}$ with a standard ${ }^{137} \mathrm{Cs}$ source.

ionic emission as noise. This noise should be reduced as much as possible by cooling the PMT. For this purpose, the PMT was cooled with a Peltier-device connected to the metal-packaged PMT. This cooling device required electric power of $7 \mathrm{~W}$, keeping the PMT at $0^{\circ} \mathrm{C}$ when operating the system at room temperature. The average background level falls to about $170 \mathrm{cps}$ when cooled, compared with a rate of $>3000$ cps without cooling.

To reduce the background counts due to the blackbody radiation, the area of the heater top was designed to be as small as possible, and stray-light produced from the heater was excluded as much as possible. For this purpose, four ceramics heaters were encapsulated into a brass frame giving a rod of $5 \mathrm{~mm}$ diameter and $5 \mathrm{~mm}$ length. These ceramic heaters are commercially available for soldering semiconductor circuits (size of one heater; $1.2 \times 7 \times 75 \mathrm{~mm})$ and require $32 \mathrm{~W}(100 \mathrm{~V})$ at maximum heating. Since the heating part is limited only to the top part, black-body radiation could be significantly reduced in comparison with the normal large Nichrome-heater plate. When a sample pan is conveyed under the photondetection part by the carousel, the sample pan on this heater rod is lifted to avoid direct heat contact between the sample pan and the carousel, followed by heating start. Since the sample pan has a biotite brim, the area exposed for photon-detection is restricted only to the inner part of the sample vessel, as mentioned in a preceding paper (Yawata and Hashimoto, 2007). Thus, the total electric power of heater part needs $128 \mathrm{~W}$ at maximum and the highest temperature can be linearly realized up to $600^{\circ} \mathrm{C}$.

Since the sensitive surface (diameter $5 \mathrm{~mm}$ ) of the PMT is small and it is situated at a relatively long distance from the luminescence sample, only poor detection efficiency was obtainable for luminescence measurement. To improve this situation, the poor light collection was overcome using a clad-rod type glass light guide (Nissei Denki Co. Ltd.), which was fixed between the luminescent sample and the PMT.
The total size of the PMT, including the Peltierdevice, of the air-cooling system is $104 \times 76 \times 56 \mathrm{~mm}$ which is smaller than the existing photon counting box in our laboratory.

\section{The LED holder}

Sixteen blue LEDs and 16 infra-red (IR) LEDs were employed for stimulation of quartz OSL and stimulation of feldspar IRSL, respectively. Only quartz OSL measurements will be described here. A DUG11 (Schøtt) filter (detection region; 300-400 nm) was placed in front of the PMT for OSL measurements. Two sheets of SC42 (FUJI PHOTO FILM Co. Ltd.) plastic filters were placed in front of each blue LED in order to remove the troublesome tail of blue light $(<400 \mathrm{~nm})$, which can cause high background due to the overlap between detection window and stimulation light wavelength.

The same LED holder can be used for both pulsedOSL (P-OSL) measurements as well as for continuous wave OSL (CW-OSL) measurements. For the P-OSL measurement, the following pulse widths and an interval time can be adjusted, pulse widths may be $2,4,8$ or $10 \mu \mathrm{s}$ and the time interval between pulses is $200-1600 \mu$ s.

In the case of P-OSL, the luminescence photon counting rate after pulsed stimulation should be accumulated as a function of time over periods of many micro-seconds. The blue LEDs were used to provide pulses $10 \mu$ s long and the P-OSL signal was measured during the subsequent 200 microsecond intervals (see Fig. 6). For the sake of such rapid pulse procedures, a fast pulse counter and on-line display system has also been employed (Yawata et al., 2006). In this system, LED trigger pulses at the ending of LED light off and luminescence pulses are fed to an ordinal 32bit PCI board (Z-cosmos, ZH-HTS2). This PCI board consists of timing control IC and counting IC. All trigger and luminescence pulse occurring time data were stored to fast in fast out (FIFO) RAM, followed by transfer into CPU RAM providing $1 \mu$ s resolution time. An accumulated P-OSL decay curve from every repeated stimulation and luminescence measurement were displayed on CRT in real-time. Finally, the P-OSL data can be obtained either in an Excel form or text format.

\section{PERFORMANCE TESTS}

\section{Sample preparation}

In the experiment, two kinds of quartz samples were used. One was extracted from an ancient roof-tile (ShinYakushiji temple, Nara, Japan, built in A.D. 747.). The equivalent dose of this quartz sample has previously been estimated using another handmade system (Nakata et al., 2007). Another sample was extracted from a volcanic ash layer (Medeshima layer, Miyagi, Japan). The quartz extraction procedure is described briefly here. All extraction procedures were carried out under faint red light to avoid any influence of light-bleaching effects on the naturally accumulated signals in quartz. The samples were gently crushed into suitable sizes in an agate mortar and washed with distilled water, $6 \mathrm{M} \mathrm{HCl}$ and $\mathrm{NaOH}$ for 1 hour and then treated with concentrated HF for 30 minutes under 
an ultrasonic agitator at $50^{\circ} \mathrm{C}$. The remaining material was subjected to density separation $\left(2.63-2.67 \mathrm{~g} / \mathrm{cm}^{3}\right)$ using sodium polytungstate solutions to purify the quartz fraction. The quartz samples were sieved to select grains of $75-150 \mu \mathrm{m}$ diameters. The volcanic quartz sample was heated at $450^{\circ} \mathrm{C}$ for 5 minutes to erase the naturally accumulated dose.

\section{Comparison between the new system and the existing system}

In order to check the usefulness of the new system for RTL measurement, RTL glow curve measurements were carried out for the estimation of the signal-to-noise ratio ( $\mathrm{S} / \mathrm{N}$ ratio) using a volcanic quartz sample, which was irradiated with a fixed dose of $50 \mathrm{~Gy}$. In this test, a combination of an R-60 (Toshiba) and an IRC-65L (Kenko) filter, giving a detection region from 600-700 nm, were employed. The preheat condition and heating rate were $220^{\circ} \mathrm{C}$ for $5 \mathrm{~min}$ and $1{ }^{\circ} \mathrm{C} / \mathrm{s}$, respectively. The samples were initially subjected to first glow curve measurement, followed by a background-curve mainly due to blackbody radiation. The background-curve was subtracted from the first glow curve. The remaining curve is called the "RTL glow curve". A RTL glow curve peak of quartz appeared at about $320^{\circ} \mathrm{C}$ as seen in Fig. 3. Both the RTL glow curve and the background-curve were integrated over the range $310-330^{\circ} \mathrm{C}$, which are called the "RTL signal" and the "Background", respectively. Identical measurements, using the same detection filters, were carried out using the present equipment and by the older systems described by Nakagawa et al. (2003).

As it is well known, RTL measurements with low background level are difficult because of the appearance of black-body radiation which starts to appear beyond $250^{\circ} \mathrm{C}$. To reduce the background to as low as level as possible when measuring the red emission, the area of the ceramics heater was minimized and tight light-shielding
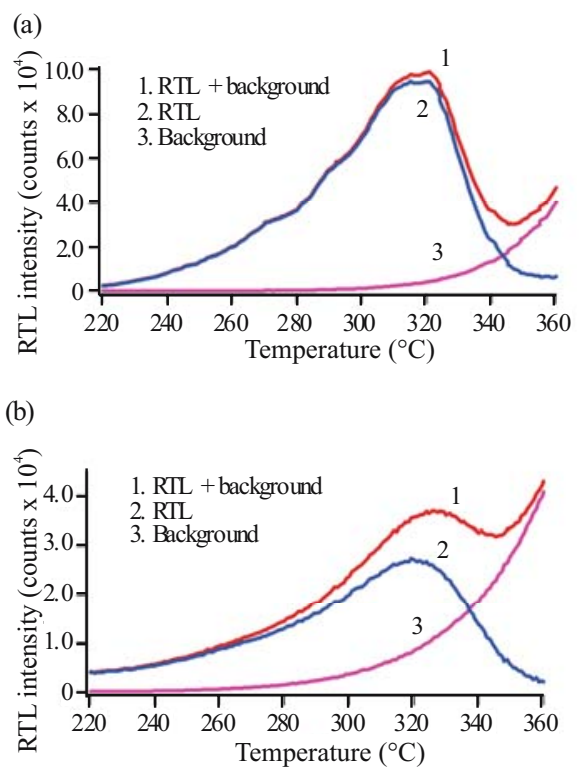

Fig. 3. Comparison of RTL measurements between (a) the new system described in the paper and (b) the existing handmade system. was used between the heater head and the inlet of the light-guide. In addition, selection of the optimal optical filter combinations was made. In the tests, the same quartz sample and the same detection filter assembly were employed for both measurement systems.

The RTL signals, background values and $\mathrm{S} / \mathrm{N}$ ratios of each system are summarized in Table 1. Table 1 shows that the new system gives a higher $\mathrm{S} / \mathrm{N}$ ratio for $\mathrm{RTL}$ signals in comparison with the existing handmade one. This means that the new system also could eliminate the thermal background counts more effectively than the existing handmade one, as seen Fig. 3 and Table 1. The new system has a lower thermal background $\left(1.9 \times 10^{4}\right.$ counts, Fig. 3a) than the older system $\left(2.5 \times 10^{4}\right.$ counts, Fig. 3b), resulting in a prominent peak in RTL at about $320^{\circ} \mathrm{C}$. This reduction is because light leakage from the heater was eliminated in the new system.

The high RTL measurement performance of the new system may be due to the high sensitivity of the metalpackaged PMT in the red-region relative to the PMT with a multi-alkaline surface used in the older system in spite of the same optical filter combinations.

\section{Equivalent dose evaluation from burnt archaeological roof-tile}

In both TL colour image (TLCI) and TL-spectrometry (Hashimoto et al. 1986, 1997), quartz samples from roof tiles showed red-TL emissions. For estimation of the RTL equivalent dose, the single-aliquot regenerative-dose (SAR) protocol was adopted using special software developed for this system. In RTL measurements, the samples were heated from $50-400^{\circ} \mathrm{C}$ at a heating rate of $1^{\circ} \mathrm{C} / \mathrm{s}$ with a preheat temperature at $240^{\circ} \mathrm{C}$ for 5 minutes. The applied X-ray dose-rate was $1.35 \mathrm{~Gy} / \mathrm{min}$ when the X-ray tube voltage and current were $30 \mathrm{kV}$ and $15 \mu \mathrm{A}$, respectively.

The RTL signals were integrated in the plateau range $\left(320-340^{\circ} \mathrm{C}\right)$, which are decided from the plateau test of TL glow curves derived from induced and natural RTL as shown in Fig. 4a and b. The RTL intensities after correction of RTL sensitivity changes, when applying a test dose of 1.35 Gy between the measurements, are plotted against the regenerative doses to construct a dose response curve as seen in Fig. 5. The equivalent dose could be estimated by interpolating the integrated natural RTL (NTL) intensity onto the dose-response curve. In these measurements, it was found from a test dose measurement that no sensitivity change occurred between each measurement of the natural or a regenerated dose.

The naturally accumulated dose in three aliquots was estimated to be $4.44 \pm 0.36 \mathrm{~Gy}$, which agreed well with the

Table 1. Comparison between the new system and the existing handmade one. These results were obtained from one measurement as shown in Fig. 3. The reproducibility of each glow curve was also ensured.

\begin{tabular}{lccc}
\hline & $\begin{array}{c}\text { RTL signal } \\
\left(\text { counts } \times 10^{4}\right)\end{array}$ & $\begin{array}{c}\text { Background } \\
\left(\text { counts } \times 10^{4}\right)\end{array}$ & S/N ratio \\
\hline New mini system & 21.8 & 1.9 & 11.4 \\
Handmade system & 7.6 & 2.5 & 3.0 \\
\hline
\end{tabular}


previous results $(4.4 \sim 5.11 \mathrm{~Gy}$, dependent on the portion of roof tile) using the handmade system (Nakata et al., 2007). The experimental error on the equivalent dose is derived from the standard deviation on the three aliquots analyzed. From this result, this system has been confirmed to be suitable for RTL measurement with high detection efficiency with a high experimental reproducibility.

\section{Preliminary pulsed OSL measurement}

An aliquot of volcanic quartz was used to demonstrate the measurement of pulsed OSL. After preheating for $5 \mathrm{~min}$. at $220^{\circ} \mathrm{C}$, the sample temperature was kept at $125^{\circ} \mathrm{C}$ during blue-light pulsed stimulation, and the emitted signal was recorded with a resolution of $1 \mu \mathrm{s}$. The applied stimulation photon pulse width is fixed to $10 \mu \mathrm{s}$ and the phosphorescence detection period (or interval measuring time) is kept at $200 \mu$ s. Fig. 6 shows a typical time-resolved curve for the volcanic quartz sample irradi-

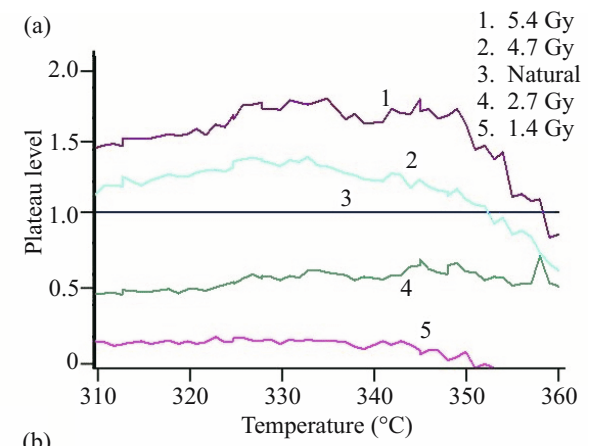

(b)

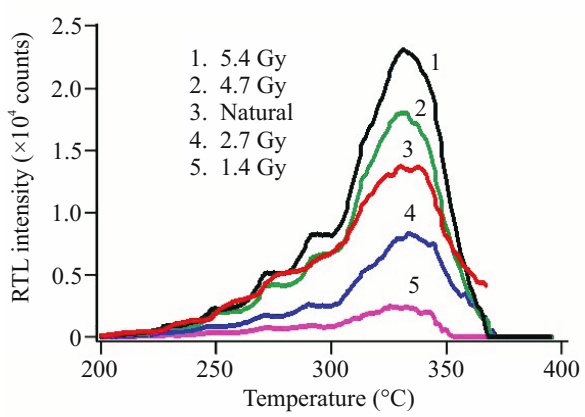

Fig. 4. Changes of RTL glow curves (a) and plateau tests (b) for quartz grains extracted from the roof tile of the Shin-Yakushiji temple.

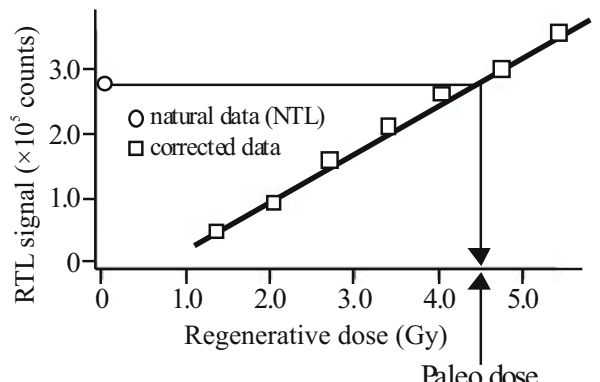

Fig. 5. RTL dose response curve from a sample of the roof tile of the Shin-Yakushiji temple. RTL sensitivity changes between the measurements were corrected on the basis of luminescence intensities when applying the test dose of $1.35 \mathrm{~Gy}$. ated by $\gamma$-ray of ${ }^{137} \mathrm{Cs}$ with $150 \mathrm{~Gy}$. The time-resolved curve shown in Fig. 6 consists of the integrated signal from $1.0 \times 10^{5}$ light pulse exposures and photon-detection interval repetitions, which corresponds to the equivalent of a $1 \mathrm{~s} \mathrm{CW-OSL} \mathrm{illumination.} \mathrm{From} \mathrm{the} \mathrm{decay-behaviour}$ analysis, the mean decay lifetime of this quartz sample was evaluated to be $40.5 \pm 1.9 \mu \mathrm{s}$. This lifetime value agrees with about $35 \mu \mathrm{s}$, which was evaluated by Chithambo and Galloway (2000) for other quartz samples by a similar P-OSL method and $40 \mu$ s as found by Denby et al. (2006). Accordingly, this decay signal could be attributed to the afterglow of the quartz sample.

\section{CONCLUSIONS}

We successfully constructed a portable mini automated luminescence-measuring system fitted with a miniature X-ray generator and metal-packaged PMT. The new system is readily movable and greatly decreases the electric power consumption compared with the existing system. The volume of the main box is small, approximately $300 \times 300 \times 300 \mathrm{~mm}$, excluding another box (about $230 \times 130 \times 350 \mathrm{~mm}$ ) containing the heater controller and $\mathrm{X}$-ray controller. An outstanding problem is the additional electric power required for the ceramic heater (128 $\mathrm{W}$ at maximum), but a gas-burner heater system combined with a small gas-reservoir may be available in the future and provide a potential solution.

Compared with the existing system, the new system improves the signal to noise ratio for RTL while offering smaller size and weight (about $15 \mathrm{~kg}$ instead of $35 \mathrm{~kg}$ in the older system) of the main steel box, including the $\mathrm{X}$-ray irradiator and photon-detection system. This makes the system portable, so that it could be used at archaeological sites and in classrooms. Moreover, P-OSL is also confirmed to be possible to use in the new system.

In the RTL dose evaluation from a burnt archaeological roof-tile, the naturally accumulated dose from the new system was in good agreement with previous results (Nakata et al., 2007). This means that the new system is useful for practical measurements of RTL-signals either for dosimetry or dating purposes with a high signal-tonoise ratio.

\section{ACKNOWLEDGEMENTS}

The authors would like to express their appreciation

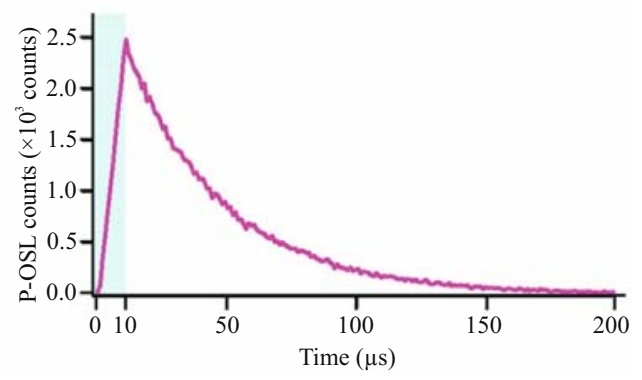

Fig. 6. Time resolved response of quartz under pulsed stimulation. The results have been integrated for $1.0 \times 10^{5}$ pulse repetitions, which corresponds to a total of $1 \mathrm{~s} \mathrm{CW-OSL} \mathrm{illumination.}$ 
to $\mathrm{Mr}$. S. Itou, Z-cosmos Co. Ltd., for providing valuable information related to data-processing and electronics for the present measurement system.

We would like to thank Professor G.A.T. Duller, University of Wales, Aberystwyth, for his valuable comments and kind corrections of our manuscript. The present work was supported greatly by Grant-in Aids for Fundamental Science Research from Ministry of Education, Culture, Sports, Science and Technology, Japan (no. 17500691)

\section{REFERENCES}

Aitken MJ, 1985. Thermoluminescence Dating. London, Academic Press: $359 \mathrm{pp}$.

Aitken MJ, 1998. An Introduction to Optical Dating. The Dating of Quaternary Sediments by the Use of Photon-stimulated Luminescence. Oxford, Oxford University Press: 267pp.

Bøtter-Jensen L, McKeever SWS, and Wintle AG, 2003. Optically Stimulated Luminescence Dosimetry. Amsterdam, Elsevier: 355pp.

Chithambo ML and Galloway RB, 2000. On luminescence lifetime in quartz. Radiation Measurements 32(5-6): 621-626, DOI 10.1016/S1350-4487(00)00096-2.

Denby PM, Bøtter-Jensen L, Murray AS, Thomsen KJ, and Moska P, 2006. Application of pulsed OSL to the separation of the luminescence components from a mixed quartz/feldspar sample. Radiation $\begin{array}{lll}\text { Measurements } & 41(7-8): & 774-779,\end{array}$ 10.1016/j.radmeas.2006.05.017.

Fattahi M and Stokes S, 2000. Red thermoluminescence (RTL) in volcanic quartz: development of a high sensitivity detection system and some preliminary findings. Ancient TL 18(2): 35-44.

Ganzawa Y, Frukawa H, Hashimoto T, Sanzelle S, Mialliere D and Pillevre J, 2005. Single grains dating of volcanic quartz from pyroclastic flows using Red TL. Radiation Measurements 39(5): 479487, DOI 10.1016/j.radmeas.2004.10.012
Hashimoto T, Koyanagi A, Yokosaka K, Hayashi Y and Sotobayashi T, 1986. Thermoluminescence color images from quartzes of beach sands. Geochemical Journal 20: 111-118.

Hashimoto T, Sugai N, Sakaue H, Yasuda K, and Shirai N, 1997. Thermoluminescence (TL) spectra from quartz grains using on-line TL spectrometric system. Geochemical Journal 31: 189-201.

Hashimoto T, Nakagawa T, Hong DG, and Takano M, 2002. An automated system for both red/blue thermoluminescence and optically stimulated luminescence measurement. Journal of Nuclear Science and Technology 39(1): 108-109, DOI 10.3327/jnst.39.108.

Murray AS and Wintle AG, 2000, Luminescence dating of quartz using an improved single-aliquot regenerative-dose protocol. Radiation Measurements 32(1): 57-73, DOI 10.1016/S1350-4487(99)00253$\mathrm{X}$.

Nakagawa T, Usuda H and Hashimoto T, 2003. Optically stimulated luminescence (OSL) and thermoluminescence (TL) measurements on red TL (RTL) quartz samples using a new automated OSL/TL measuring system. Journal of Radioanalytical and Nuclear Chemistry 255(2): 355-358, DOI 10.1023/A:1022517123226.

Nakata Y, Tamaki M, and Hashimoto T, 2007. Red thermoluminescence dating using quartz grains extracted from a roof-tile of an old Japanese temple. Journal of Radioanalytical and Nuclear Chemistry 272(2): 433-438, DOI 10.1007/s10967-0070542-1.

Poolton NRJ, Bøtter-Jensen L, Wintle AG, Jakobsen F and Knudsen KL, 1994. A portable system for the measurement of sediment OSL in the field. Radiation Measurements 23(2-3): 529-532, DOI 10.1016/1350-4487(94)90093-0.

Yawata T and Hashimoto T, 2004. Availability of a small X-ray tube combined with $\mathrm{Al}$-absorber as a new irradiator for luminescence researches. Radioisotopes 53: 207-212.

Yawata T, Sakaue H, Itou S and Hashimoto T, 2006. Usability of a new multiple high speed pulse time data registration, processing and realtime display system for pulse time interval analysis. Transactions of the Atomic Energy society of Japan 5: 221-228 (in Japanese).

Yawata T and Hashimoto T, 2007. Development of a TL detection system for a single grain of quartz. Radiation Measurements 42(9): 1460-1468, DOI 10.1016/j.radmeas.2007.03.008. 